\title{
Neural Tube Defects
}

a

0. 


\section{Neural Tube Defects}

\section{Jorge A Lazareff}

University of California Los Angeles, USA 


\section{Published by}

World Scientific Publishing Co. Pte. Ltd.

5 Toh Tuck Link, Singapore 596224

USA office: 27 Warren Street, Suite 401-402, Hackensack, NJ 07601

UK office: 57 Shelton Street, Covent Garden, London WC2H 9HE

\section{British Library Cataloguing-in-Publication Data}

A catalogue record for this book is available from the British Library.

\section{NEURAL TUBE DEFECTS}

Copyright (C) 2011 by World Scientific Publishing Co. Pte. Ltd.

All rights reserved. This book, or parts thereof, may not be reproduced in any form or by any means, electronic or mechanical, including photocopying, recording or any information storage and retrieval system now known or to be invented, without written permission from the Publisher.

For photocopying of material in this volume, please pay a copying fee through the Copyright Clearance Center, Inc., 222 Rosewood Drive, Danvers, MA 01923, USA. In this case permission to photocopy is not required from the publisher.

ISBN-13 978-981-4273-84-8

ISBN-10 981-4273-84-8

Typeset by Stallion Press

Email: enquiries@stallionpress.com

Printed in Singapore. 


\section{This page is intentionally left blank}


To Barbara van de Wiele, M.D. 


\section{This page is intentionally left blank}




\section{Contents}

1. Introduction 1

2. Pathogenesis 8

3. Classification 25

$\begin{array}{ll}\text { Part I: Spinal Defects } & 27\end{array}$

4. Myelomeningocele 29

5. Occult Spinal Bifida 92

6. Cervical Spinal Dysraphism 98

$\begin{array}{ll}\text { 7. Lipomyelomeningocele } & 102\end{array}$

8. Meningocele 122

9. Hemimyelomeningocele 127

10. Diastematomyelia 129

11. Terminal Myelocystocele 144

12. Caudal Regression Syndrome 149

13. Dorsal Enteric Fistula 155

14. Segmental Spinal Dysgenesis Syndrome 160

15. Neurenteric Cyst 165

16. Dermal Sinus 168

17. Thickened Filum Terminal Syndrome 172 
18. Tethered Cord Syndrome 176

19. Syringomyelia 193

Part II: Cranial Defects 207

20. Anencephaly 209

21. Iniencephaly 215

22. Encephalocele 220

23. Hydrocephalus 257

24. Multiple NTD 263

25. Genetic Syndromes with Neural Tube Defects 266

$\begin{array}{ll}\text { Glossary } & 270\end{array}$

$\begin{array}{ll}\text { Index } & 279\end{array}$ 